\title{
$i$-BRUSH: A Gaze-Contingent Virtual Paintbrush for Dense 3D Reconstruction in Robotic Assisted Surgery
}

\author{
Marco Visentini-Scarzanella ${ }^{1}$, George P. Mylonas ${ }^{1,2}$, Danail Stoyanov ${ }^{2}$, \\ and Guang-Zhong Yang ${ }^{1,2}$ \\ ${ }^{1}$ Royal Society/Wolfson Foundation MIC Laboratory \\ ${ }^{2}$ Institute of Biomedical Engineering, \\ Imperial College London, London SW7 2AZ, United Kingdom \\ \{marco.visentini-scarzanella02, george.mylonas, danail.stoyanov, \\ g.z.yang\} @imperial.ac.uk
}

\begin{abstract}
With increasing demand on intra-operative navigation and motion compensation during robotic assisted minimally invasive surgery, real-time 3D deformation recovery remains a central problem. Currently the majority of existing methods rely on salient features, where the inherent paucity of distinctive landmarks implies either a semi-dense reconstruction or the use of strong geometrical constraints. In this study, we propose a gaze-contingent depth reconstruction scheme by integrating human perception with semi-dense stereo and $p-q$ based shading information. Depth inference is carried out in real-time through a novel application of Bayesian chains without smoothness priors. The practical value of the scheme is highlighted by detailed validation using a beating heart phantom model with known geometry to verify the performance of gaze-contingent 3D surface reconstruction and deformation recovery.
\end{abstract}

\section{Introduction}

In robotic assisted minimally invasive surgery (MIS), the ability to reconstruct 3D tissue deformation in real-time is required for the prescription of dynamic active constraints [1], motion stabilisation, and intra-operative image guidance. Recently, it has been shown that it is possible to recover the 3D shape and morphology of soft-tissue surfaces using a stereo-laparoscope for anatomical regions with distinctive appearance. However, to recover 3D structure from homogeneous tissue areas with limited texture details, strong geometrical constraints such as hierarchical piecewise bilinear maps, B-splines and thin-plate splines have been used to provide dense disparity maps [2-4]. With these approaches, surface discontinuities and specular highlights are significant problems as is the assumption that the scene is composed of a single smooth surface. Alternative techniques based on photometric algorithms forgo the need for explicit feature mapping: by assuming an a priori physical image formation model, particularly the co-axial arrangement of the endoscope camera and light-source, dense $3 \mathrm{D}$ reconstruction is feasible. The uniqueness of viscosity solutions to a HamiltonJacobi equation for the perspective projection case can be established but most methods assume uniform albedo and a Lambertian reflection model [5]. Systems integrating multiple visual cues through inference over Markov Random Fields 
(MRF) for 3D reconstruction have been demonstrated [6, 7]. However, their computational complexity limits their applicability in scenarios requiring real-time performance and high precision.

Recently, the concept of gaze-contingent perceptual docking through binocular eye tracking has attracted extensive interest [8]. Current results suggest that 3D recovery and motion stabilisation in the fovea is sufficient, while stabilisation in the periphery does not affect user and visual perceptual quality [9]. The strength of the gaze-contingent method is that it relies on the innate ability of the human vision, independently from the geometrical characteristics of the scene presented.

The purpose of this paper is to present an interactive gaze-contingent approach to dynamically reconstruct the 3D structure of the surgeon's area of interest. Surface recovery is formulated as an inference problem combining stereo disparity estimation and surface shading for increased robustness. Computational complexity is limited by considering only the surgeon's area of interest, and by a novel usage of Bayesian chains instead of MRFs for propagation without smoothness constraints. A fast regularisation scheme ensures all information available is used without loopy propagation, enabling real-time operation.

The proposed method, called $i$-BRUSH (Bayesian Reconstruction Using Stereo and sHading), is validated on a da Vinci surgical robot using a beating heart phantom with known geometrical and temporal ground truth data for surface reconstruction and tissue deformation recovery.

\section{Method}

The proposed method consists of four distinct processes. First, the surgeon's fixation point is localised on the left and right channels of the input video stream from the endoscope through the use of a binocular eye-tracker fully integrated with the da Vinci surgical robot's master console. The eye-tracker consists of a pair of near infrared sensitive cameras, an array of externally switchable miniaturised IR emitting diodes at $940 \mathrm{~nm}$ and a pair of dichroic beam splitters with cut-off wavelength at $750 \mathrm{~nm}$. This allows both eyes to be tracked at $50 \mathrm{fps}$ after a short eye calibration procedure as outlined in the work by Mylonas et al. [10].

Then, stereo reconstruction is employed to provide a semi-dense estimation of the surface within the small area of high visual acuity corresponding to roughly 3 to 5 degrees of visual angle. This is achieved by using a Shi-Tomasi detector followed by pyramidal Lucas-Kanade tracker for matching. This sparse 3D information is then locally propagated, yielding a semi-dense reconstruction. Independently from the stereo reconstruction process, local surface orientation expressed as the surface directional gradient vectors $\left(p_{i}, q_{i}\right)$ is estimated for each pixel $m_{i}$ by approximating the image irradiance equation based on Lambertian reflection and the geometrical constraints of the endoscope camera and light-sources as described in [11].

The final stage of the proposed method focuses on fusion of the semi-dense stereo map with the $p-q$ surface gradient to obtain a dense reconstruction of the area. To this end, a Bayesian inference scheme is used. While a grid network would have to be solved through approximate methods, thus introducing excessive computational burden, a polytree is used instead for efficient inference at each image element. This 


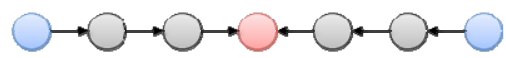

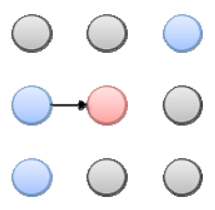

(a)

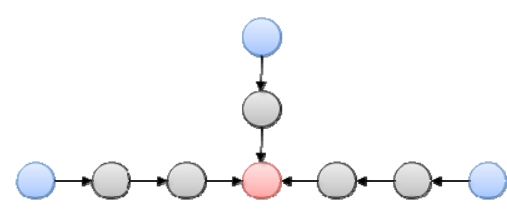

(c) (b)

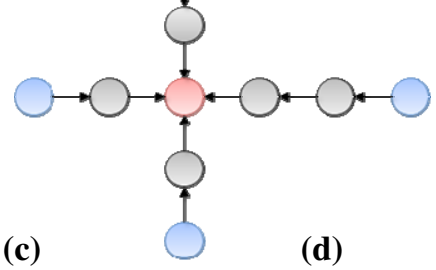

Fig. 1. Network topologies depending on the number of stereo-reconstructed points present along the horizontal and vertical directions. Stereo reconstructed points are shown in blue, the point to be inferred in red and points with only $p-q$ information are shown in grey. (a) The network contains only one stereo point along the horizontal or vertical direction; (b) two points along the same direction; (c) three chains; and (d) four chains, respectively. The chains may not be symmetrical as the first stereo pixel along a given direction is taken.

structure consists of a maximum of four Bayesian chains in a star configuration, each starting from a separate stereo-reconstructed point and terminating at the pixel to be inferred. These Bayesian chains represent the shortest direct path between the point to be inferred and the stereo-reconstructed points, with the terminating points imposing depth constraints and the nodes along the chain providing the natural encoding of the $p-q$ gradient derived from local surface shading. Four different topologies are possible depending on the number of stereo points, and these are illustrated in Fig. 1.

The problem of exact inference along causal polytrees, such as the ones illustrated in Fig. 1, is well studied and requires a computationally intensive Belief Propagation (BP) message passing scheme. It can be shown that without the use of smoothness constraints, the problem can be reduced to a simple recursive scheme.

Let us define the problem of finding the Maximum A Posteriori (MAP) estimate of the distance from the camera centre $D_{k}$ for the $k^{\text {th }}$ element along one chain, with upstream information being propagated from $N$ chains. The information propagated consists of $\Delta D^{n+}$ and $p q^{n+}$, as the depth gradient and $p$ - $q$ information propagated from the $n^{\text {th }}$ upstream chain. Since a node is conditionally independent on all others given its own Markov blanket, the MAP estimate can be written as:

$$
\tilde{D}_{k}=\arg \max \left\{P^{\prime}\left(D_{k}\right)\right\}=\underset{D_{k} \in \mathbb{R}}{\arg \max }\left\{P\left(D_{k} \mid \Delta D_{k-1}^{n+}, p q_{k}, p q_{k-1}^{n+}\right)\right\}
$$

Separating upstream and element evidence, and setting $\alpha$ as a normalisation constant:

$$
P^{\prime}\left(D_{k}\right)=\alpha\left(P\left(D_{k} \mid p q_{k}\right) \prod_{n^{+} \in N} P\left(D_{k} \mid \Delta D_{k-1}^{n+}, p q_{k-1}^{n+}\right)\right)
$$

which can be solved with belief propagation requiring likelihood information to be passed to all nodes along the chain. Since $D_{k}=D_{k-1}+\Delta D_{k}$, (2) can be rewritten as:

$$
P^{\prime}\left(D_{k}\right)=\alpha\left(P\left(\Delta D_{k} \mid p q_{k}\right) \prod_{n^{+} \in N} P\left(\Delta D_{k} \mid \Delta D_{k-1}^{n+}, p q_{k-1}^{n+}\right)\right)+\beta \sum_{n^{+} \in N} D_{k-1}^{n+}
$$


The normalisation constant $\beta$ is equal to the reciprocal of the number of chains. As the surface gradient at the $k^{\text {th }}$ node is independent from its neighbours' gradient -unless surface smoothness is assumed- the gradient can be inferred from the likelihood relationship with the instantiated value of the surface gradient:

$$
P^{\prime}\left(D_{k}\right)=P\left(\Delta D_{k} \mid p q_{k}\right)+\beta \sum_{n^{+} \in N} D_{k-1}^{n+}
$$

The recursion can be expanded up to the terminal points of the chain, which propagate the initial stereo values. The MAP estimate of the gradient can then be obtained from likelihood data correlating surface gradient and $p-q$ values combined with depth information from the terminal nodes. The matrices are obtained for each image frame by discretising the range of values, obtained from the stereo and $p-q$ measurements, into a number of bins sufficient for maintaining precision while guaranteeing realtime performance.

Local inaccuracies in the $p-q$ and stereo reconstructions are mitigated by the use of global per-frame statistics, however, errors in the instantiated values during inference can result in a cumulative error. By assuming that the error at each step is normally distributed, the estimation error of $\tilde{D}_{k}$ can be reduced by setting it as the weighted average of the estimates $D_{k-1}^{n+}$ from the $N$ converging chains. The weighting factor is determined by the number of nodes $K_{n}$ along the chain as a fraction of the total number of nodes $K$ from all chains. Thus, for the point to be inferred, the normalisation constant $\beta$ can be set as a weighted average accordingly, yielding:

$$
\tilde{D}_{k}=\arg \max \left\{P\left(\Delta D_{k} \mid p q_{k}\right)+\frac{1}{Z} \sum_{n^{+} \in N} \frac{K-K_{n}}{K} D_{k-1}^{n+}\right\}
$$

The normalisation constant $1 / Z$ ensures the weights add up to one. While (5) can be used to recover the depth of the point $m_{i}$ to be inferred, it does not address how to use the other estimates obtained along the chains. Such information can be used to update the local frame statistics if it is regularised to reflect the new weighted average estimate of $m_{i}$. To regularise the estimates without explicit smoothness constraints, the chain can be modelled as a spring series fixed at one end. The stiffness constant of each spring is inversely proportional to the number of nodes in the chain, since the confidence of the estimate decreases with each inferential step, and the onedimensional relaxed position of the $k^{\text {th }}$ spring joint along the $n^{\text {th }}$ chain is equal to its MAP estimate. The difference between the weighted average and the estimate for each chain $\Delta m_{i}^{n}$ can be then represented as the result of a constant force $F$ acting on the spring system. The force is distributed along the springs according to their relative stiffness constants, where the compressed position of the $k^{\text {th }}$ spring joint is equal to the new depth estimate at the corresponding node along the chain. The force is calculated from the displacement of a single equivalent spring:

$$
F=x_{e q} p_{e q}=\Delta m_{i}^{n}\left(\sum_{k=1}^{K_{n}} \frac{1}{p_{k}}\right)^{-1}=\Delta m_{i}^{n}\left(\sum_{k=1}^{K_{n}} k\right)^{-1}
$$


Since the force is constant at each joint, the displacement of each node along the $n^{\text {th }}$ chain $\Delta m_{k}^{n}$ can be calculated by equating the force on the equivalent spring with the force at each joint and solving for $\Delta m_{k}^{n}$. The likelihood matrices for the elements along each inference chain are then updated to integrate the new evidence for subsequent iterations involving any subset of the inferred nodes. Once all points not previously reconstructed have been inferred, their depth is converted into $3 \mathrm{D}$ coordinates by projecting a ray of length equal to the estimated depth from the camera centre through the $2 \mathrm{D}$ point $m_{i}$ on the retinal plane.

\section{Experimental Setup and Results}

To evaluate the accuracy of the proposed depth reconstruction scheme, a silicone phantom heart (Chamberlain Group, MA, USA) has been recorded at $25 \mathrm{fps}$ using the stereo laparoscope from a da Vinci system integrated with the 3D binocular eyetracker as described earlier. To facilitate the registration process during validation, the phantom heart was augmented with 15 silicone fiducials visible on its surface and scanned with a Siemens Somatom Sensation 64 CT scanner. The image resolution was $0.41 \times 0.41 \times 0.5 \mathrm{~mm}$ and the temporal resolution was $0.33 \mathrm{~s}$. In this experiment, the heart rate was set to $90 \mathrm{bpm}$, yielding twenty $3 \mathrm{D}$ volumes covering the entire cardiac cycle. To ensure the accuracy of the ground truth data, the cardiac volumes and the fiducials were segmented interactively and the spatial coordinates of the fiducials were tracked. An image of the phantom together with a 3D rendering of one cardiac phase indicating the location of the fiducials is shown in Fig. 2.

Two sets of experiments were performed to illustrate the potential applications of the proposed method. First, the operator was asked to fixate on the surface of the static phantom describing continuous paths in order to demonstrate the method's accuracy in
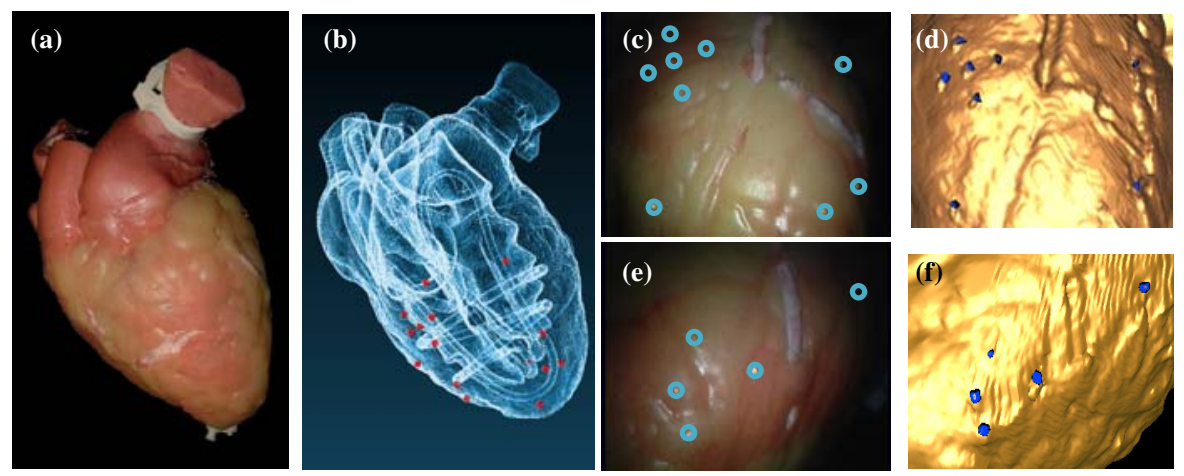

Fig. 2. (a) The heart phantom and (b) its corresponding 3D rendering from the 4D CT data. Fiducial locations are highlighted in red on the epicardial surface. (c), (e) Image frames from LAD and left ventricle sequences, fiducials highlighted in light blue. (d), (f) Registration of ground truth data to camera coordinates of (c), (e) respectively using manually-tracked fiducial locations. 
recovering 3D information for large smooth featureless areas. Second, the operator was required to perform fixations on different regions of the epicardial surface of the beating heart phantom to evaluate the accuracy of real-time dynamic motion recovery.

For each experiment, two video sequences were recorded from different viewpoints, the first focusing on the left ventricle and the second surrounding the midsegment of the left anterior descending artery (LAD) The global pose alignment of the heart with the CT data for validation was based on the fiducials located on the epicardial surface. To calculate the optimal registration between the two coordinate systems knowing the correspondence between fiducials, the absolute orientation method by Horn [12] was used. The average alignment error was estimated to be of $1.30 \mathrm{~mm}$ and $1.18 \mathrm{~mm}$ for the sequences (c) and (e) shown in Fig. 2.

Static Surface Reconstruction. For the static sequences, paths of different lengths were visually delineated on the epicardial surface, both on uniform areas and on parts of the surface presenting distinctive landmarks. To estimate the performance numerically, the reconstruction error for each $3 \mathrm{D}$ point $\mathbf{M}_{\mathbf{i}}$ is given as the distance between $\mathbf{M}_{\mathbf{i}}$ and the closest point belonging to the ground truth set lying along the ray passing through $\mathbf{M}_{\mathbf{i}}$ and the camera origin. To this end, a ray-casting method has been implemented to recover the barycentric coordinates of the intersection point between the ray and a triangular facet described by three vertices on the CT volume. In Fig. 3, the paths reconstructed are overlaid on the original image frames together with a graphical representation of the error distribution for each frame.

It is evident that the frequencies in Fig. 3(b) and (e) show that most reconstruction errors lie in the range $0-3.5 \mathrm{~mm}$ with the outliers present in some frames significantly affecting the standard deviation of the error shown in Figs. 3(c) and 3(f). The outliers occur due to occasional drifts between the recorded left and right fixations producing incorrect stereo matches. However, as a significant proportion of the reconstructed area consists of overlapping regions between consecutive frames, the impact of outliers can be easily removed by averaging of all the estimates obtained for a given point. The average error for the overall reconstructed surfaces in Figs. 3(a) and (d) is of $2.30 \mathrm{~mm}$ and $2.03 \mathrm{~mm}$ respectively, with a standard deviation of 2.51 and $3.64 \mathrm{~mm}$. Such values are in line with the initial alignment error estimated in the previous section, suggesting the potential of the method for accurate dense $3 \mathrm{D}$ estimation of static scenes.

Dynamic Depth Recovery. The proposed method was also applied to motion estimation on the beating phantom. Evaluation was performed as previously indicated with the temporally aligned and spatially registered CT data. The accuracy of the reconstructed area was in line with that of the static experiments. The per pixel error appears to be uniformly distributed between positive and negative values, yielding an estimated average distance closer to the ground truth. Discrepancies between ground truth and recovered motion are a consequence of the inability to visually track fast moving objects with small displacements, and it would be alleviated at lower beat rates. A summary of the overall error assessment is presented in Table 1. 

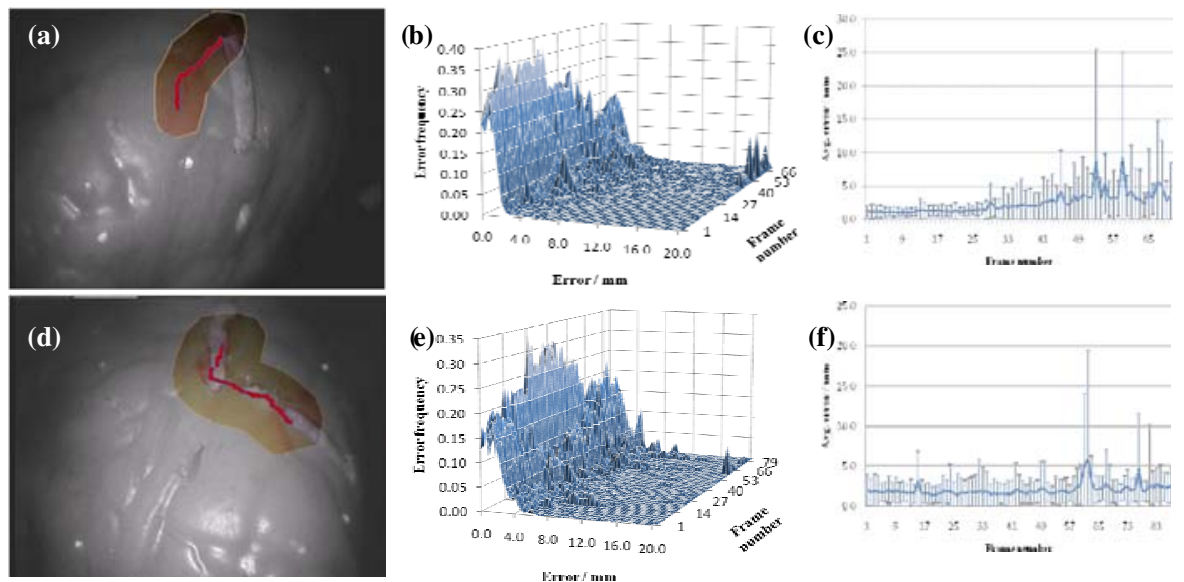

Fig. 3. (a) Left ventricle and (d) LAD section sequences, the traces of fixations along paths are shown in red. (b), (e) Per-frame reconstruction error frequency of (a), (d). (c), (f) Average and standard deviation of reconstruction error for each frame from (a), (d).
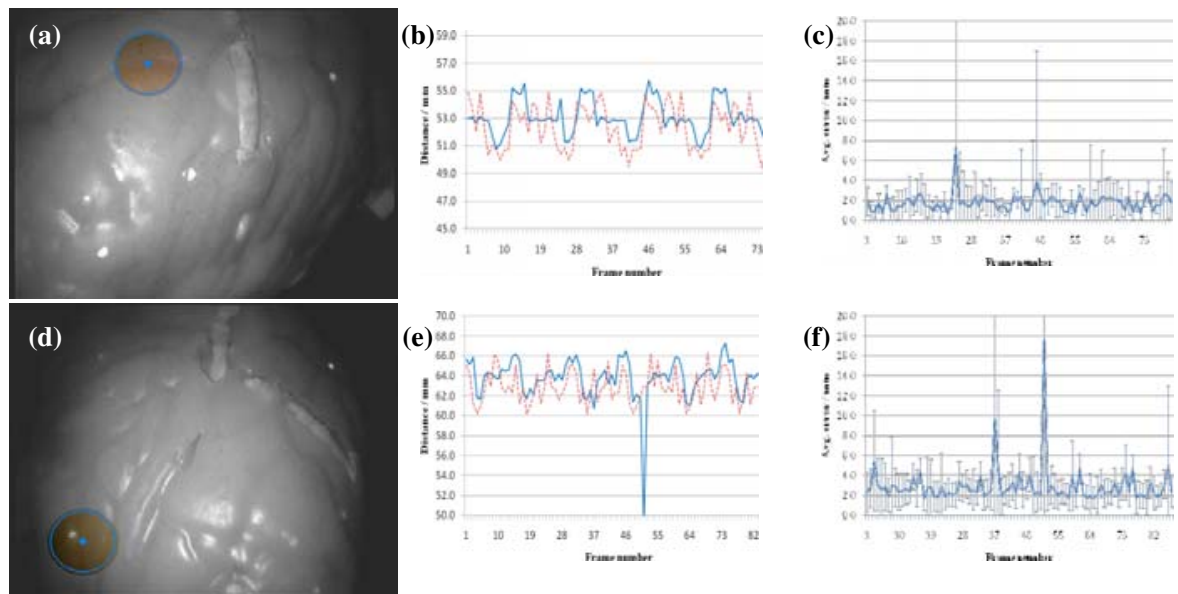

Fig. 4. (a) Left ventricle and (d) LAD sequences, the fixation area is highlighted in blue. (b), (e) Per-frame average depth of the reconstructed and ground truth fixation area. (c), (f) Perframe average error and standard deviation.

Table 1. Summary of results for static and dynamic sequences

\begin{tabular}{lccccc}
\hline \multicolumn{1}{c}{ Sequence } & $\begin{array}{c}\text { Length } \\
\text { (frames) }\end{array}$ & $\begin{array}{c}\text { Avg. distance } \\
(\mathbf{m m})\end{array}$ & $\begin{array}{c}\text { Avg. error } \\
(\mathbf{m m})\end{array}$ & $\begin{array}{c}\text { Standard } \\
\text { deviation }\end{array}$ & $\begin{array}{c}\text { Avg. error } \\
(\%)\end{array}$ \\
\hline Left ventricle (static) & 70 & 53.493 & 2.302 & 2.509 & 4.30 \\
Left ventricle (dynamic) & 80 & 53.081 & 1.809 & 2.036 & 3.41 \\
LAD (static) & 85 & 67.837 & 2.029 & 3.641 & 2.99 \\
LAD (dynamic) & 100 & 63.793 & 2.936 & 2.331 & 4.60 \\
\hline
\end{tabular}




\section{Discussion and Conclusions}

In this paper, we have presented a new framework for intra-operative 3D reconstruction from multiple depth cues for addressing the problem of dense reconstruction without imposing prior geometric constraints. The reconstructed surface is limited to the surgeon's main area of interest, thus facilitating real-time implementation with no detrimental effect on perceptual quality. It has been further demonstrated that with the proposed method the removal of explicit smoothness constraints has no adverse effect on the reconstruction accuracy while reducing computational complexity. Experimental results from a phantom model with known ground truth highlight the value of the technique for both static and dynamic scenes, with potential applications in image guidance, motor channelling and motion compensation. Future work will explore the inclusion of multiple reflectance models to better represent the material properties and temporal cues for added robustness.

\section{References}

1. Mylonas, G., Kwok, K.W., Darzi, A., Yang, G.-Z.: Gaze-Contingent Motor Channelling and Haptic Constraints for Minimally Invasive Robotic Surgery. In: Metaxas, D., Axel, L., Fichtinger, G., Székely, G. (eds.) MICCAI 2008, Part II. LNCS, vol. 5242, pp. 676-683. Springer, Heidelberg (2008)

2. Lau, W.W., Ramey, N.A., Corso, J.J., Thakor, N.V., Hager, G.D.: Stereo-Based Endoscopic Tracking of Cardiac Surface Deformation. In: Barillot, C., Haynor, D.R., Hellier, P. (eds.) MICCAI 2004. LNCS, vol. 3217, pp. 494-501. Springer, Heidelberg (2004)

3. Richa, R., Poignet, P., Liu, C.: Efficient 3D Tracking for Motion Compensation in Beating Heart Surgery. In: Metaxas, D., Axel, L., Fichtinger, G., Székely, G. (eds.) MICCAI 2008, Part II. LNCS, vol. 5242, pp. 684-691. Springer, Heidelberg (2008)

4. Stoyanov, D., Darzi, A., Yang, G.-Z.: Dense 3D Depth Recovery for Soft Tissue Deformation During Robotically Assisted Laparoscopic Surgery. In: Barillot, C., Haynor, D.R., Hellier, P. (eds.) MICCAI 2004. LNCS, vol. 3217, pp. 41-48. Springer, Heidelberg (2004)

5. Prados, E., Faugeras, O.: Shape from shading: a well-posed problem? In: IEEE Computer Society Conference on CVPR, vol. 872 (2005)

6. Yuille, A., Kersten, D.: Vision as Bayesian inference: analysis by synthesis? Trends in Cognitive Sciences 10(7), 301-308 (2006)

7. Lo, B.P.L., Visentini Scarzanella, M., Stoyanov, D., Yang, G.-Z.: Belief Propagation for Depth Cue Fusion in Minimally Invasive Surgery. In: Metaxas, D., Axel, L., Fichtinger, G., Székely, G. (eds.) MICCAI 2008, Part II. LNCS, vol. 5242, pp. 104-112. Springer, Heidelberg (2008)

8. Mylonas, G.P., Darzi, A., Yang, G.-Z.: Gaze Contingent Depth Recovery and Motion Stabilisation for Minimally Invasive Robotic Surgery. In: Yang, G.-Z., Jiang, T.-Z. (eds.) MIAR 2004. LNCS, vol. 3150, pp. 311-319. Springer, Heidelberg (2004)

9. Mylonas, G., Stoyanov, D., Darzi, A., Yang, G.-Z.: Assessment of Perceptual Quality for Gaze-Contingent Motion Stabilization in Robotic Assisted Minimally Invasive Surgery. In: Ayache, N., Ourselin, S., Maeder, A. (eds.) MICCAI 2007, Part II. LNCS, vol. 4792, pp. 660-667. Springer, Heidelberg (2007)

10. Mylonas, G.P., Darzi, A., Yang, G.-Z.: Gaze-contingent control for minimally invasive robotic surgery. Computer Aided Surgery 11(5), 256-266 (2006)

11. Rashid, H.U., Burger, P.: Differential algorithm for the determination of shape from shading using a point light source. Image Vision Comput 10(2), 119-127 (1992)

12. Horn, B.K.P.: Closed-form solution of absolute orientation using unit quaternions. J. Opt. Soc. Am. A 4(4), 629-642 (1987) 